\section{NLRC4: panic button for the calm intestinal phagocyte}

It is now well appreciated that phagocytes that inhabit the intestine have a phenotype quite distinct from those of macrophages and dendritic cells isolated or generated from the spleen, peritoneal cavity, or bone marrow. Perhaps the starkest difference is that intestinal phagocytes are in a state of "inflammatory anergy" in that they do not produce proinflammatory mediators in response to Toll-like receptor ligands but, rather, serve to promote tolerance to commensal bacteria and food antigens. However, recent findings have shown that these "peace-loving" cells are in fact equipped with the immune system's most powerful and highly controlled danger signal-inflammasome-mediated production of interleukin (IL)-1 $\beta$-and they use it to protect against invasive flagellated pathogens such as Salmonella typhimurium.

Effective use of a hierarchal defense system requires maintaining appropriate control of communications. In the immune system, this concept manifests in the relative abilities of various cells to produce cytokines that control the movement and activation state of various populations of immune cells. For example, whereas most cells (including epithelial, endothelial, and other nonimmune cells) are able to produce chemokines that drive localized immune cell recruitment, fewer cell types are able to generate "master" proinflammatory cytokines such as IL-12 and tumor necrosis factor- $\alpha$ that have more dramatic and systemic effects on host defense and physiology. The use of the single-most potent cytokine-IL-1 $\beta$, which can dramatically alter host physiology at the cellular and organismal levels - is further restricted not only by tight control over licensing of its synthesis but by requiring activation of an entirely distinct signaling pathway - the inflammasome-to process pro-IL-1 $\beta$ into the mature active cytokine. ${ }^{1}$

Moreover, in contrast to transcriptionally controlled cytokine production, which can be triggered merely upon extracellular detection of microbial products, inflammasome activation requires intracellular recognition of select microbial components, thus limiting induction of IL-1 $\beta$ to situations in which bacteria become intracellular. Such multilayered control over II-1 $\beta$ ought to minimize unwarranted development of fever and other systemic responses driven by IL-1 $1 \beta$ without blocking the ability of cells to call for limited immune cell recruitment.

Although such multilayered control of II- $1 \beta$ production might seem sufficient to protect against inappropriate triggering of systemic inflammation in most locales, given the enormous bacterial load in the intestine, one might think a logical safeguard might be to not arm intestinal cells with inflammasome machinery altogether. Indeed, intestinal phagocytes (a collective term for intestinal dendritic cells and macrophages, which are not readily distinguished from each other by classic markers), in stark contrast to other populations of macrophages and dendritic cells, are unresponsive to Toll-like-receptor agonists. ${ }^{2}$ Such hyporesponsiveness is thought to be a mechanism to prevent inappropriate triggering of inflammation. Rather, intestinal phagocytes voraciously consume bacteria and are thought to play a key role in promoting tolerance to such bacteria largely via their constitutive production of anti-inflammatory cytokines such as IL-10 (ref. 3). However, recent work by Franchi and colleagues ${ }^{4}$ shows that, although these cells were not deemed trustworthy to call for local security, they are equipped to activate the ultimate danger signal of inflammasome-mediated IL-1 $\beta$ production. Specifically, the authors observed that intestinal phagocytes were unresponsive to Toll-like-receptor agonists but produced copious levels of mature IL-1 $\beta$ ex vivo or in vivo in response to pathogenic Salmonella typhimurium. Such IL-1 $\beta$ production required expression of flagellin by the bacteria and host expression of the NLRC4 inflammasome, which is known to recognize flagellin. The fact that activation of intestinal phagocyte NLRC4 required intracellular flagellin was demonstrated by a requirement of a Salmonella type 3 secretion system, which mediates invasion, and by the ability to overcome this requirement by using the detergent streptolysin-O.

To use a military analogy, giving the intestinal phagocyte (previously considered to be in a state of inflammatory anergy) the ability to produce IL- $1 \beta$ is comparable to preventing a field soldier from radioing his base but enabling him to alert the entire army if he felt the situation warranted it. Moreover, in contrast to all other known cells, intestinal phagocytes store preformed IL-1 $\beta$, removing the first layer of control of expression of this powerful cytokine. The mechanisms that regulate expression and storage of such preformed pro-IL- $1 \beta$ remain to be defined, but they are not dependent on recognition of microbes because intestinal phagocytes from germ-free mice still stored pro-IL-1 $\beta$. In any case, intestinal phagocytes not only have the ability to disseminate a red alert but are actually poised-and seemingly eagerto do so.

The fact that intestinal phagocytes can activate the NLRC4 to alter host defense/ physiology would seem to provide substantial benefit in the event of infection by flagellated pathogens, and, indeed, mice deficient in the NLRC4 inflammasome exhibit increased susceptibility to oral Salmonella infections ${ }^{5}$ (although the extent of the increase is mouse-strain-dependent). However, equipping the intestinal phagocyte with the immune system's ultimate alarm signal would seem to invite the possibility of inappropriate inflammation. Given the strong association of elevated inflammasome cytokines, namely, IL-1 $\beta$ and IL-18, with seemingly inappropriate states of inflammation, particularly in inflammatory disease, this fear is indeed too frequently realized. Nonetheless, given that phagocytes 
in the intestine are clearly playing by a number of different rules, it is perhaps wise to presume that NLRC4 is but one small piece of a puzzle we are only beginning to understand.

\section{Andrew T Gewirtz}

C 2012 Society for Mucosal Immunology

\section{REFERENCES}

1. Dinarello, CA. IL-1: discoveries, controversies and future directions. Eur. J. Immunol. 40, 599606 (2010).

2. Smythies, L.E. et al. Human intestinal macrophages display profound inflammatory anergy despite avid phagocytic and bacteriocidal activity. J. Clin. Invest. 115, 66-75 (2005).

3. Denning, T.L., Wang, Y.C., Patel, S.R., Williams, I.R. \& Pulendran, B. Lamina propria macrophages and dendritic cells differentially induce regulatory and interleukin 17-producing T cell responses. Nat. Immunol. 8, 1086-1094 (2007).

4. Franchi, L. et al. NLRC4-driven production of $\mathrm{IL}-1$ beta discriminates between pathogenic and commensal bacteria and promotes host intestinal defense. Nat. Immunol. 13, 449-456 (2012).

5. Carvalho, F.A. et al. Cytosolic flagellin receptor NLRC4 protects mice against mucosal and systemic challenges. Mucosal Immunol. 5, 288-298 (2012). 